\title{
Revisión General de Isquemia Mesentérica Aguda, Clasificación, Diagnóstico y Manejo.
}

\section{General Review of Acute Mesenteric Ischemia, Classification, Diagnosis and Management.}

Pág. 75,84

Dr. Ignacio Bolaños Alvarado. Médico general. Médico independiente, Costa Rica

Dr. Angélica Castillo Gutiérrez. Médico general. Médico independiente, Costa Rica

Dr.Shamila Kourbanov Steller. Médico general. Médico independiente, Costa Rica
Recibido: 20-04-2020

Aceptado: 27-05-2020

\section{Resumen}

La isquemia mesentérica aguda se caracteriza por una reducción de flujo sanguíneo a nivel intestinal, lo que lleva a clasificarlo como una emergencia cuyo diagnóstico es un reto clínico y precisa de un tratamiento precoz.

Es una patología que se presenta de manera más frecuente en la población adulta mayor, alcanzando un porcentaje de presentación que va desde el 0.09 al $0.2 \%$ de la población general.

Existe una amplia gama de presentación de síntomas donde predomina el dolor abdominal súbito en epigastrio o mesogastrio. En el momento de la sospecha clínica se debe considerar la estabilidad del paciente para que de esta manera se aborde con métodos diagnósticos recomendados.

El manejo debe incluir la descompresión gastrointestinal; reanimación con fluidoterapia agresiva para corregir la hipovolemia, el soporte hemodinámico y la monitorización, además de la corrección electrolítica y ácido-base.

Palabras Clave: Isquemia mesentérica aguda, isquemia mesentérica, tratamiento endovascular.

Abstract: Acute mesenteric ischemia (AMI) is characterized by a reduction in blood flow at the intestinal level, so it seeks to classify it as an emergency whose diagnosis is a clinical review and requires early treatment.

It is a pathology that occurs more frequently in the older adult population, reaching a percentage of presentation that ranges from 0.09 to $0.2 \%$ of the general population. There is a wide range of symptom presentation where sudden abdominal pain in epigastrium or mesogastrium predominates. At the time of clinical suspicion, the stability of the patient should be considered so that this is addressed with the recommended diagnostic methods.

Management should include gastrointestinal decompression; resuscitation with aggressive fluid therapy to correct hypovolemia, hemodynamic support and monitoring, in addition to electrolyte and acid-base correction.

Key Words: Acute mesenteric ischemia, mesenteric ischemia, endovascular treatment.

\section{Introducción}

La isquemia mesentérica aguda (IMA) ha sido un reto diagnóstico y terapéutico a lo largo de la historia. 
Para superar los desafíos relacionados al diagnóstico, en primera instancia se debe reconocer que esta patología no es rara, sino una condición común en la población adulta mayor.

Además, la fisiopatología es compleja y existe una presentación clínica muy variada, por lo que es de vital importancia conocer a cabalidad estos dos puntos.

A pesar de que en las últimas décadas la Tomografía Axial Computarizada (TAC) con contraste se ha convertido en uno de los exámenes diagnósticos más importantes en pacientes que ingresan al servicio de emergencias con dolor abdominal agudo en sospecha de IMA, hay que tener en consideración que los signos en TAC en las primeras etapas de la patología suelen ser sutiles y difíciles de detectar, por lo que la clave del diagnóstico siempre será la sospecha clínica, ya que el diagnóstico y tratamiento temprano ha demostrado que más de la mitad de los pacientes pueden ser rescatados.

Otras estrategias como la trombolisis dirigida por catéter ha ayudado en el manejo de esta patología. (1)

En la revisión se abordaran factores epidemiológicos, tipos de isquemia mesentérica y factores de riesgo asociados a la entidad etiopatogénica, técnicas diagnósticas, fases de la enfermedad, manejo inicial y tratamiento. Recordar que hablamos de una enfermedad con una compleja fisiopatología y una alta morbimortalidad por lo que los conocimientos, orden y fundamentos científicos, son esenciales para mejorar la sobrevida.

\section{Métodos}

La selección de artículos para esta revisión bibliográfica, se encontraron artículos en inglés y español, con fecha de publicación desde el 2014 al 2020. Se realizaron las búsquedas en bases de datos como PubMed, MedLine, UpToDate y buscadores como Google Scholar. Para ello se utilizaron términos de búsqueda como "Isquemia mesentérica aguda", "Mesenteric ischemia", además de "Isquemia mesentérica" combinado con "diagnóstico", "manejo", "tratamiento".

Posteriormente, de los artículos obtenidos, se filtraron los de mayor importancia científica. Se revisaron los resúmenes, resultados, y en casos necesarios, los artículos completos; seleccionando así, finalmente, los documentos que incluían la información necesaria para el cumplimiento de los objetivos de dicha revisión bibliográfica.

\section{Epidemiología}

La IMA es una patología rara en pacientes jóvenes, generalmente es considerada una enfermedad del adulto mayor, ya que afecta principalmente a los pacientes mayores de 60 años, siendo su incidencia directamente proporcional al aumento de edad y existiendo predominio del sexo masculino (2).

Alcanza una incidencia anual desde el 0.09 al $0.2 \%$, siendo de $1-2 \%$ de los abdómenes agudos $(3,4)$.

Generalmente es considerada una enfermedad del adulto mayor, ya que afecta principalmente a los pacientes mayores de 60 años, siendo su incidencia directamente proporcional al aumento de edad y existiendo predominio del sexo masculine (2).

La tasa de mortalidad está estrechamente relacionada al grado de necrosis isquémica intestinal (2). A pesar de los avances y técnicas de laboratorio se asocia a una mortalidad mayor del $60 \%$ si se retrasa el diagnóstico en $12 \mathrm{~h}$ (5).

\section{Anatomía}

El suministro arterial de los intestinos está dado por 3 arterias principales que son ramas de la Aorta Abdominal; la arteria celíaca, la arteria mesentérica 
superior (AMS) y la arteria mesentérica inferior (AMI), éstas se interconectan para proteger al intestino de periodos de hipoperfusión gracias a la circulación colateral que existe entre ellas.

La AMS es la arteria principal ya que irriga el intestino delgado y la primera mitad del colon, mientras que la AMI perfunde el resto del colon y recto, por lo que una oclusión aguda de la AMS podría ser devastadora $(3,6)$.

\section{Fisiopatología}

La fisiopatología de la isquemia mesentérica es compleja y las manifestaciones clínicas bastante diversas, lo que dificulta el diagnóstico temprano (2).

El desarrollo de la lesión isquémica en el intestino se produce al existir una inadecuada perfusión, con insuficiente suministro de oxígeno y aporte de nutrientes; al existir una reducción prolongada en el flujo sanguíneo va a conducir a vasoconstricción en el lecho vascular afectado y eventualmente reduce el flujo sanguíneo colateral $(5,6)$

Con el transcurso de la isquemia, se produce una disrupción de la barrera intestinal ocasionada por la liberación de mediadores químicos, motivada por la traslocación bacteriana generando una necrosis difusa de la mucosa extendiéndose a la pared abdominal. Resultando en un infarto transmural, perforación, necrosis, sepsis y falla multiorgánica ${ }^{2}$

\section{Clasificación}

La isquemia mesentérica aguda puede clasificarse en: Embolia aguda arterial, Trombosis arterial aguda, Trombosis venosa mesentérica e Isquemia mesentérica no oclusiva. Cada una con sus factores de riesgo asociados (ver tabla-1).
1. Embolia aguda arterial:

Es la causa más frecuente de isquemia mesentérica, en un $40-50 \%$ de los casos. El émbolo suele alojarse en la AMS $(3,7,8)$. El origen puede incluir la aurícula izquierda en pacientes con fibrilación auricular (FA) $(70 \%)$, el ventrículo izquierdo en casos de trombo mural ventricular y de aneurisma aórtico, además un $20 \%$ de estos pacientes pueden presentar embolismo arterial periferico $(4,7)$.

\section{Trombosis arterial aguda:}

Representa el $20 \%$ de los casos de isquemia mesentérica (4). Se presenta en pacientes con aterosclerosis de larga data quienes pueden desarrollar una placa más frecuente a nivel de AMS, seguido por el tronco celíaco y la AMI (4) y producir un flujo sanguíneo turbulento con la subsecuente estenosis lo cual producirá un dolor posprandial, conocido como "angina intestinal" y "miedo a la comida" que resultará en una pérdida de peso, se ve ne un $80 \%$ de los pacientes con isquemia mesentérica crónica (3).

\section{Trombosis venosa mesentérica:}

Representa del $5-15 \%$ de los casos $(3,8)$. La edad media de presentación es de 45-60 años, con un ligero predominio femenino (4). Suelen padecerla los pacientes con estados de hipercoagulabilidad como se ve en: hipertensión portal, toma de anticonceptivos, trombofilia, trauma, cambios inflamatorios locales (pancreatitis, diverticulitis) $(3,7,8)$.

Estos pacientes presentan síntomas menos severos, pero más insidiosos que los que presentan oclusión arterial. Suelen padecerla los pacientes con estados de hipercoagulabilidad como se ve en: hipertension portal, toma de anticonceptivos, trombofilia, trauma, cambios inflamatorios locales (pancreatitis, diverticulitis)

\section{(9) $(1) \Theta \theta$}




\begin{tabular}{|c|c|c|c|}
\hline $\begin{array}{l}\text { Embolismo } \\
\text { arterial. }\end{array}$ & $\begin{array}{l}\text { Trombosis } \\
\text { arterial. }\end{array}$ & $\begin{array}{l}\text { Trombosis venosa } \\
\text { mesentérica. }\end{array}$ & $\begin{array}{l}\text { Isquemia } \\
\text { mesentérica } \\
\text { no oclusiva. }\end{array}$ \\
\hline $\begin{array}{l}\text { Edad } \\
\text { avanzada. } \\
\text { Tabaquismo. } \\
\text { HTA. } \\
\text { Cardiopatía. } \\
\text { Hiperlipidemia. } \\
\text { DM. } \\
\text { ERC. } \\
\text { AV. } \\
\text { Válvula } \\
\text { protésica } \\
\text { cardiaca. } \\
\text { Enfermedad } \\
\text { reumática } \\
\text { cardiaca. } \\
\text { FA. } \\
\text { IAM. }\end{array}$ & $\begin{array}{l}\text { HTA. } \\
\text { Hiperlipidemia. } \\
\text { DM. } \\
\text { Estrógenos. } \\
\text { Ateroesclerosis. } \\
\text { Síndrome } \\
\text { antifosfolipídico. } \\
\text { Deshidratación. } \\
\text { Estado } \\
\text { procoagulante. } \\
\text { Bajo gasto } \\
\text { cardiaco. }\end{array}$ & $\begin{array}{l}\text { ICC derecha. } \\
\text { TVP. } \\
\text { Cirrosis. } \\
\text { Hepato- } \\
\text { esplenomegalia. } \\
\text { Sepsis. } \\
\text { Enfermedad de } \\
\text { células falciforme. } \\
\text { Malignidad. } \\
\text { Pancreatitis. } \\
\text { Hepatitis. } \\
\text { Desórdenes } \\
\text { procoagulantes. } \\
\text { Hipertensión portal. } \\
\text { Trauma abdominal } \\
\text { directo. } \\
\text { Estado } \\
\text { posoperatorio. } \\
\text { Obesidad. } \\
\text { Embarazo. }\end{array}$ & $\begin{array}{l}\text { Edad }>70 \text { años. } \\
\text { Enfermedad } \\
\text { crítica. } \\
\text { Bajo gasto } \\
\text { cardiaco. } \\
\text { Enfermedad } \\
\text { vascular } \\
\text { periférica. } \\
\text { IR. } \\
\text { Tratamiento } \\
\text { con diuréticos. } \\
\text { Cirugía } \\
\text { cardiaca. } \\
\text { Mal estado } \\
\text { cardiaco } \\
\text { preoperatorio. } \\
\text { Bypass } \\
\text { cardiopulmonar } \\
\text { urgente y/o } \\
\text { extenso. }\end{array}$ \\
\hline
\end{tabular}

Tabla-1. Factores de riesgo en IMA, según su etiología. Hipertensión arterial (HTA), Diabetes mellitus (DM), Enfermedad renal crónica $(\mathrm{ERC})$, Aneurisma ventricular (AV), Fibrilación auricular (FA), Infarto de miocardio (IAM), Insuficiencia cardíaca (ICC), Trombosis venosa profunda (TVP), Insuficiencia renal (IR), Ventilación Mecánica Asistida (VMA). Adaptado de Lawson RM. Mesenteric Ischemia. Crit Care Nurs Clin North Am [Internet]. 2018;30(1):29-39. Available from: https://doi.org/10.1016/j. cnc.2017.10.003.

\section{Clínica}

La presentación clínica de la isquemia mesentérica es muy inespecífica y varía según la causa, sin embargo, el dolor abdominal es el síntoma presente en la mayoría de casos. La forma de aparición más común es súbito, localizado en epigastrio o mesogastrio con tendencia al dolor intenso, con la característica que a la palpación no existe sensibilidad objetiva, además, generalmente se acompaña con diarrea o vómitos $(3,10)$.

Los síntomas pueden variar, desde un rápido

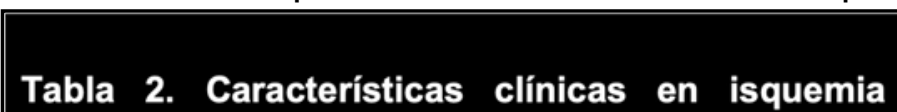

\section{mesentérica}

\begin{tabular}{|l|l|}
\hline Clasificación & Clínica \\
\hline $\begin{array}{l}\text { Embolia } \\
\text { arterial }\end{array}$ & $\begin{array}{l}\text { Inicio súbito dolor abdominal, } \\
\text { vómitos, diarrea, distensión, } \\
\text { taquicardia }\end{array}$ \\
\hline $\begin{array}{l}\text { Trombosis } \\
\text { arterial }\end{array}$ & $\begin{array}{l}\text { Dolor abdominal postprandial } \\
\text { progresivo, náuseas, pérdida de } \\
\text { peso }\end{array}$ \\
\hline $\begin{array}{l}\text { Trombosis } \\
\text { venosa }\end{array}$ & $\begin{array}{l}\text { Inicio insidioso, dolor abdominal } \\
\text { vago }\end{array}$ \\
\hline No oclusiva & $\begin{array}{l}\text { Dolor abdominal creciente, agudo, } \\
\text { estado mental alterado }\end{array}$ \\
\hline
\end{tabular}

Tabla-1. Factores de riesgo en IMA, según su etiología. Hipertensión arterial (HTA), Diabetes mellitus (DM), Enfermedad renal crónica $(E R C)$, Aneurisma ventricular $(\mathrm{AV})$, Fibrilación auricular (FA), Infarto de miocardio (IAM), Insuficiencia cardíaca (ICC), Trombosis venosa profunda (TVP), Insuficiencia renal (IR), Ventilación Mecánica Asistida (VMA). Adaptado de Lawson RM. Mesenteric Ischemia. Crit Care Nurs Clin North Am [Internet]. 2018;30(1):29-39. Available from: https://doi.org/10.1016/j. cnc.2017.10.003. 


\section{Diagnóstico}

El primer paso al presentarse un paciente con características clínicas sugestivas de isquemia mesentérica es definir la estabilidad del mismo. En pacientes estables se deben realizar estudios de analítica, radiografía de abdomen simple y/o ecografía.

La parte analítica no es concluyente, ya que no existe una prueba de laboratorio específica; lo hallazgos incluyen marcadores hematológicos de rutina como leucocitosis con desviación a la izquierda en el $75 \%$ y acidosis metabólica en un $50 \%(12)$.

Puede encontrarse además elevación de deshidrogenasa láctica (DHL), creatininafosfocinasa (CPK), fosfatasa alcalina (FA), dímero $\mathrm{D}$, sin embargo, han demostrado una muy baja tasa de especificidad (2).

Nuevos biomarcadores como I-FABP (Proteína de la Unión de Ácidos Grasos Intestinales) aunque no están ampliamente disponibles han demostrado resultados prometedores para diagnóstico, pero se necesitan más investigación para establecer su precisión. ${ }^{2}$

En cuanto a las pruebas de imagen la radiografía simple puede ser normal hasta en $25 \%$ de casos y presentar datos inespecíficos comunes a otras enfermedades, sin embargo, son útiles para descartar las mismas (4).

En pacientes estables el test de elección es la angiografía por TAC, permitiendo valorar localización y extensión de la afectación intestinal $y$ de vasos, sin embargo, es costoso y poco práctico, no está ampliamente disponible y por otro lado su utilización consume tiempo, el cual
En pacientes inestables o con datos de abdomen peritonítico deben de ser intervenidos directamente mediante laparotomía, se llega al diagnóstico de isquemia mesentérica aguda hasta en $20 \%$ (10). (ver algortimo-1).

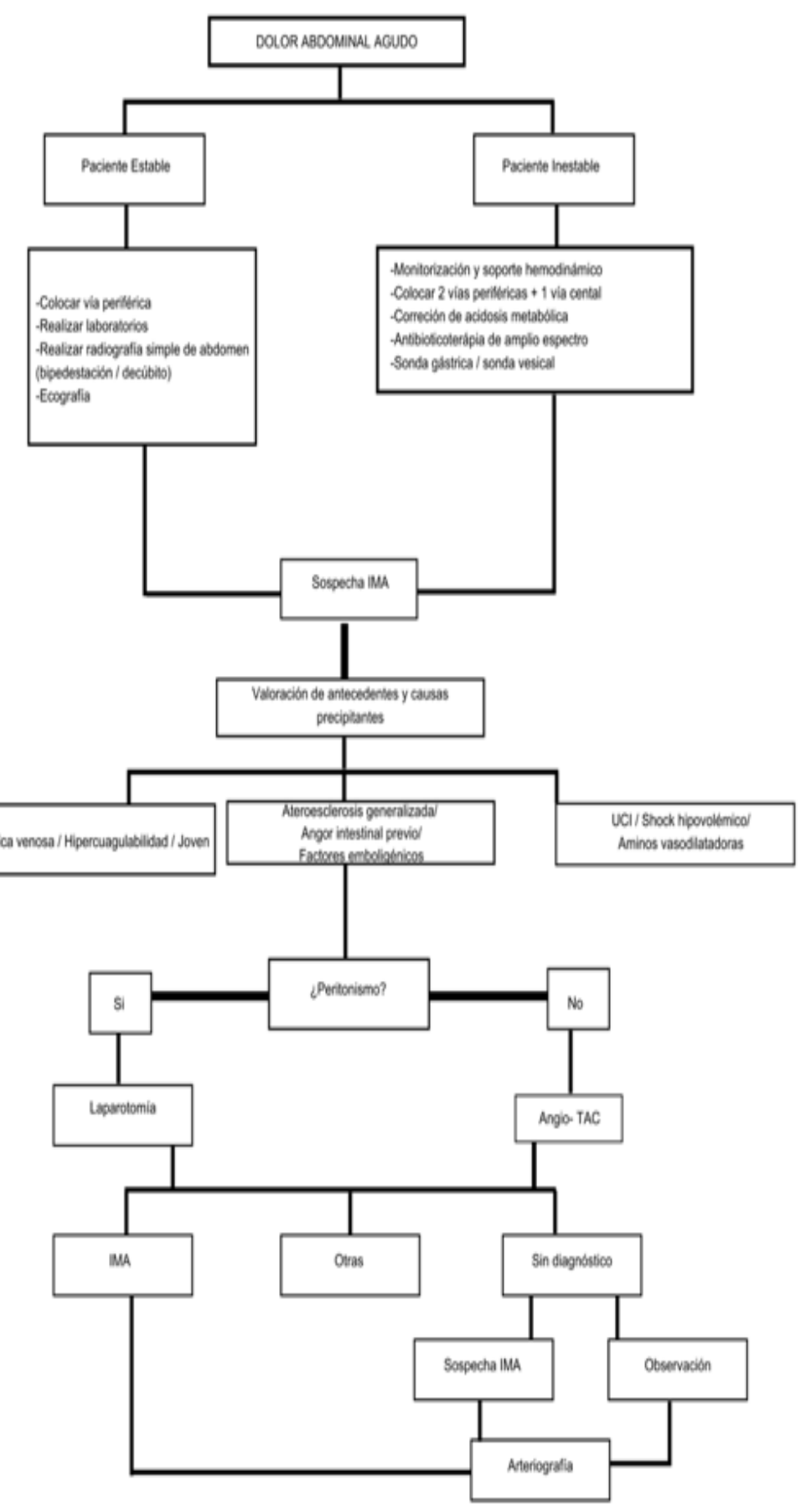

Algoritmo 1. Diagnóstico y manejo inicial de IMA. Adaptado de Herrero M, Agúndez I. Mesenteric ischaemia: Diagnosis and therapeutic algorithms. Angiologia [Internet]. 2017;69(1):34-40. Available from: http://dx.doi.org/10.1016/j.angio.2016.06.002 


\section{Fases}

Se describen 3 fases de la isquemia mesentérica: fase hiperactiva, fase paralítica y fase de shock.

En la fase hiperactiva se presenta un dolor abdominal el cual es desproporcionado, puede estar acompañado de vómito, diarrea e incluso heces sanguinolentas aunque estas últimas no siempre están presentes. La emesis y diarrea temprana son causadas por la isquemia de las capas intestinales más profundas. Al progresar la isquemia se presenta la fase paralítica, donde se presenta disminución de los movimientos intestinales y distensión abdominal; se podría detectar a la auscultación abdominal ausencia de sonidos intestinales. En la fase de shock se evidencia fuga de líquido a través de la pared intestinal que conlleva a peritonitis difusa, esto debido a la necrosis. Sin embargo, la peritonitis sólo se reporta en el $16 \%$ de los pacientes con intestino necrótico (3).

\section{Diagnóstico diferencial}

El dolor abdominal es un síntoma común a muchas patologías, por lo cual para el clínico establecer un diagnóstico definitivo es un reto.

Dentro de los diagnósticos diferenciales para isquemia mesentérica aguda se encuentran: apendicitis aguda, perforación de víscera hueca, aneurisma de aorta abdominal, colecistitis aguda y diverticulitis aguda (13).

Desde el inicio de la entrevista con los datos del paciente se puede orientar las posibilidades diagnosticas; edad, sexo y factores de riesgo brindan información de suma importancia. Posteriormente una historia clínica detallada, indagando en las características del dolor y un examen físico minucioso nos termina guiando hacia la patología más probable $(13,14)$

A partir de estos datos se puede solicitar exámenes complementarios para la confirmación de nuestra sospecha diagnostica (13).

\section{Manejo}

Elmanejoinicialsedebedehacercomoencualquier caso de abdomen agudo, inicialmente consta de identificar al paciente hemodinamicamente estable versus el inestable, en pacientes inestables se debe mantener una presión venosa central entre 8 a $12 \mathrm{mmHg}$, una presión arterial media (PAM) mayor o igual a $65 \mathrm{mmHg}$ y una diuresis superior a $0.5 \mathrm{ml} / \mathrm{kg} / \mathrm{h}$ (14). En el contexto de IMA se debe tener en cuenta: la descompresión gastrointestinal; reanimación con fluidoterapia agresiva que corrija la hipovolemia y evite la hipoperfusión secundaria a la vasoconstricción, siempre evitando la sobrecarga de volumen; el soporte y monitoreo hemodinámico; la corrección electrolítica y ácido-base; todos estos son puntos cruciales en el manejo inicial dado en las salas de shock de un servicio de emergencias, a esto se le suma el control del dolor típicamente con opioides parenterales, anticoagulación en la mayoría de los casos e inicio de antibioticoterapia de amplio espectro para prevenir y tratar la translocación bacteriana ocurrida por la isquemia de la pared $(2,8,15)$.

Agentes vasoconstrictores y digitálicos deben ser evitados debido a que exacerban el cuadro de isquemia mesentérica. Se prefiere su uso en últimas consecuencias, de ser requiera la necesidad de vasopresores, dobutamina, bajas dosis de dopamina o milrinona se prefieren debido a su menor efecto en la perfusión mesentérica comparado con otros vasopresores $(8,15)$.

La anticoagulación sistémica y agentes vasodilatores son otro pilar importante en el abordaje de la isquemia mesentérica aguda, con oclusión arterial o venosa, o no oclusivos; para así prevenir la formación y/o progresión del trombo y tratar el vaso espasmo $(8,15)$. Respecto a la anticoagulación si el paciente se encuentra con sangrado activo, como en la colitis isquémica relacionada con isquemia no oclusiva, 
la terapia no debe de ser utilizada (15). Para aquellos que requieren exploración abdominal, la anticoagulación es típicamente continuada después de la intervención quirúrgica para prevenir la formación de nuevos trombos (15).

El reposo digestivo debe ser implementado ya que se ha visto que la dieta oral puede exacerbar la isquemia aguda (8).

Pacientes sin datos de irritación peritoneal, se ven más beneficiados de una terapia de revascularización endovascular, guiada por imágenes radiologicas es centros con disponibilidad de la misma, ya que se ve una dismunución en la la morbimortalidad, gracias al rápido retorno del flujo y de tecnicas menos invasivas $(8,16)$.

La exploración abdominal quirúrgica no debe de ser retrasada independientemente de su etiología en pacientes que se sospeche por parámetros clínicos, de imagen o de laboratorio hipoperfusión severa, que con lleva a necrosis transmural, que así mismo progresa a sepsis, peritonitis, aire libre intraabdominal o una gangrena extensa. Aquellos pacientes con isquemia mesentérica no oclusiva, la exploración debe limitarse en a pacientes con signos peritoneales (15).

La meta terapéutica tiene que ir enfocada en la revascularización siempre y cuando la clínica del paciente lo permita, teniendo en cuenta la etiología, duración de la clínica y comorbilidades del paciente $(8,10,17)$.

En el embolismo arterial, el tratamiento radica en laparotomía con embolectomía si tiene signos peritoneales, cuando no presente resolución posterior a trombolisis y confirmado por estudios imagen o persiste con signos de intestino no viable. (ver algoritmo-2) El procedimiento se prefiere principalmente en pacientes con un émbolo solitario, proximal a la AMS (15). Una vez más con los nuevos avances en la medicina el abordaje multidisiplinario endovascular pueder presentar mejores resultados si la clinica del paciente lo permite (7).

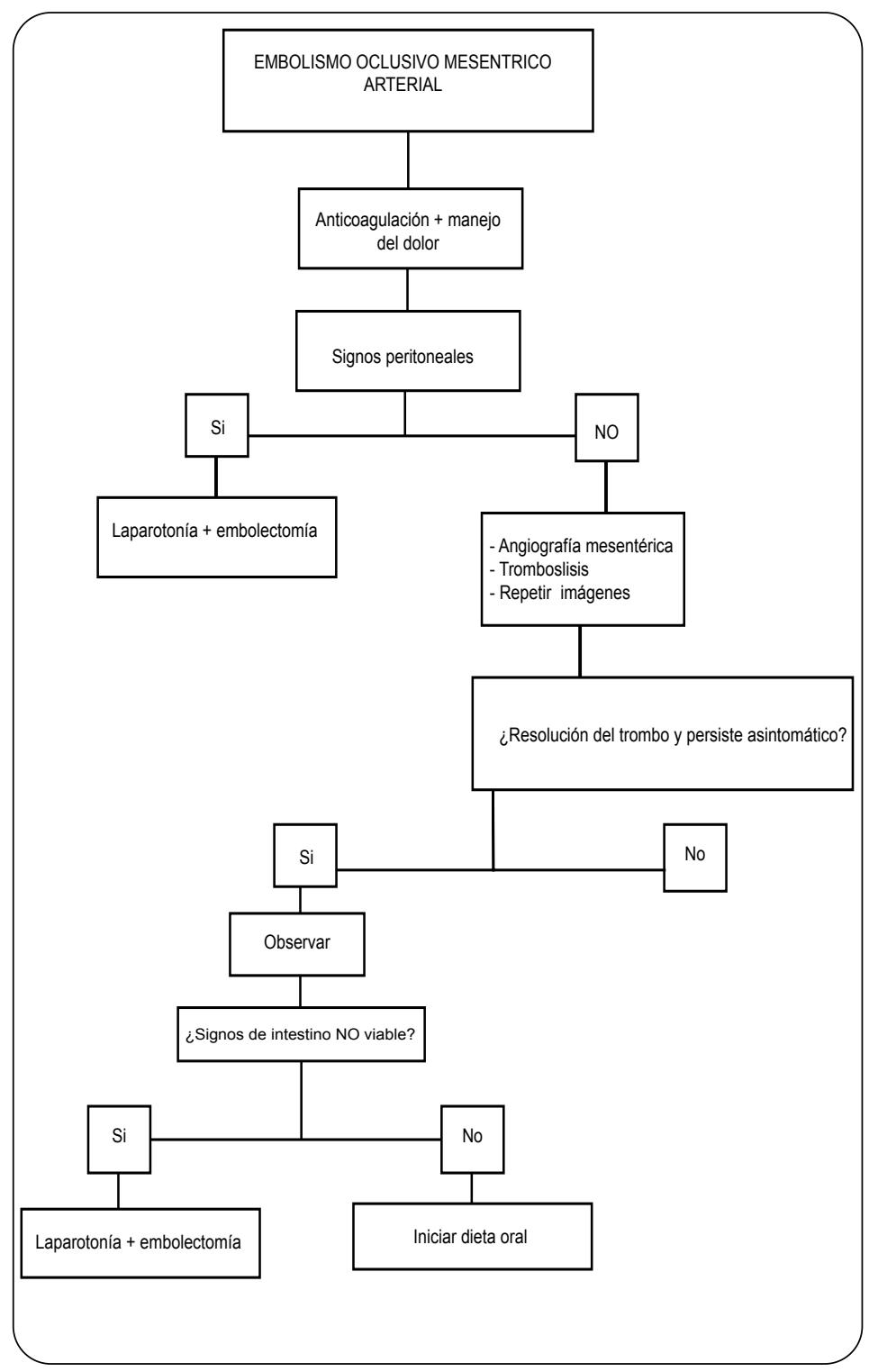

Algoritmo 2. Tratamiento de embolismo arterial mesentérico adpatado de David A Tendler, MDJ Thomas Lamont M. Overview of intestinal ischemia in adults. UpToDate [Internet]. 2018; Available from:

https://www.uptodate.com/contents/overview-of-intestinal-

ischemia-in-adults?search=isquemia mesentérica aguda\&source=search_result\&selectedTitle=1 150\&usage_ type=default\&display_rank=1\#H1822159668 
La trombosis mesentérica, la anticoagulación va a ser necesaria para todos los pacientes. Sin embargo, en aquellos que persisten con síntomas, la trombolisis puede estar indicada. Si los síntomas progresan, la exploración abdominal va a ser necesaria para para evaluar tejido intestinal no viable. (ver algoritmo-3) (15).

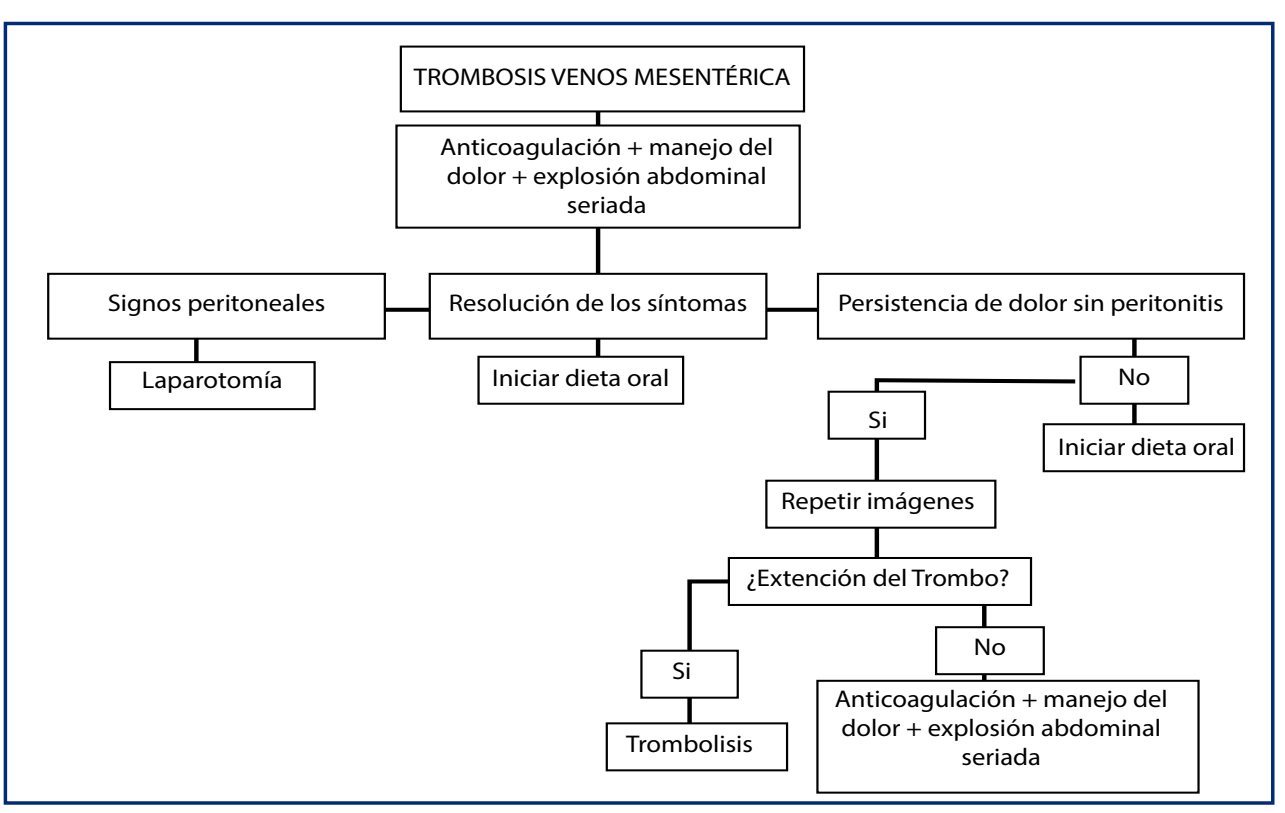

Algoritmo 3. Tratamiento de trombosis mesenterica adaptado de David A Tendler, MDJ Thomas Lamont

M. Overview of intestinal ischemia in adults. UpToDate [Internet]. 2018; Available from: https:// www.uptodate.com/contents/overview-of-intestinal-

En la isquemia no oclusiva, el tratamiento va enfocado en remover factores desencadenantes de hipotensión (medicamentos vasoconstrictores) y tratamiento de causas subyacentes (falla cardiaca, sepsis), hasta alcanzar una resolución de los síntomas de 1-2 días. Si es necesario se va a requerir soporte y monitoreo hemodinámico e infusión intraarterial de vasodilatadores. De igual manera paciente que presente síntomas peritoneales va requerir de laparotomía $(7,15)$.

En cada procedimiento quirúrgico que se realice el cirujano deberá valorar la viabilidad del tejido intestinal y necesidad de resección del mismo.
En la mayoría de los casos se prefiere realizar un cierre temporal para realizar una segunda laparotomía y así una reevaluación del tejido, y así valorar cierre e inicio de dieta oral o un retraso en el cierre abdominal (15).

\section{Complicaciones}

Dentro de las complicaciones que se pueden presentar en la IMA se pueden encontrar las que vemos en procedimientos como en la angiografía (hematomas o pseudoaneurisma); alergia o lesión renal debida al medio de contraste.

Otras complicaciones no relacionadas a la angiografía varían según la causa y tratamiento brindado en pacientes con IMA, como complicaciones peri $y$ postquirúrgicas, sepsis e infección de sitio quirúrgico; otras asociadas al uso de medicamentos como vasodilatadores (hipotensión), complicaciones asociadas a trombólisis (sangrado en sitio de acceso, embolización y accidente cerebrovascular) y lesiones a vasos periféricos durante cualquier procedimiento invasivo .

\section{Pronóstico y Mortalidad}

Comoya se ha descrito anteriormente la IMAesuna patolgía poco frecuente con una alta mortalidad, tiene una incidencia que se ve aumentada con el envejecimiento, que así mismo va de la mano con factores de riesgo cardiovasculares. Su sobrevida depende de dos pilares: el diagnóstico $y$ tratamiento precoz (16).

La sobrevida es dependiente de la agresividad con la que se maneje al paciente y comorbilidades del mismo, por lo que debe considerarse individualmente y reconocer cuando el tratamiento 
agresivo es inútil y está destinado a provocar un sufrimiento innecesario (1).

Debido a que la mayoría de los pacientes con IMA son adultos mayores y poseen distintas comorbilidades, la supervivencia a largo plazo es pobre independientemente de la eficacia del tratamiento. Los pacientes más frágiles y gravemente enfermos deben tratarse sólo con la atención adecuada, sin aumentar los riesgos (1). Es por todo lo anterior que la mortalidad según los reportes de la literatura está dentro del rango de más del $50 \%$ al $96 \%$, acercándose la mayoría al $90 \%(7,8,16)$. También, hay evidencia reciente que el abordaje endovascular está asociado con disminución de la mortalidad, además se ha visto que los pacientes no obtienen beneficio en cuanto a morbimortalidad si se intervienen quirúrgicamente en primera instancia $(6,13,15)$.

\section{Conclusiones}

La IMA es una patología de baja prevalencia, pero posee una alta morbimortalidad, además de que puede significar alto costo económico al centro de salud. Por esta razón, tener siempre la sospecha clínica es fundamental.

En todo paciente mayor de 60 años, con dolor abdominal agudo, localizado en epigastrio o mesogastrio que tuvo una instauración súbita, acompañado de vómitos y/o diarrea en algunos casos y que además viene acompañado de un rápido deterioro del paciente debe considerarse la IMA como dignóstico y realizarse las respectivas pruebas para confirmarse.

Nuevas herramientas diagnósticas que están en investigación actualmente como biomarcadores como I-FABP, pueden ayudar al clínico a realizar un adecuado diagnóstico.

A pesar de existir nuevas técnicas menos invasivas como lo es el procedimiento endovascular que ha demostrado mejorar la sobrevida de los pacientes portadores de IMA, la resolución quirúrgica sigue siendo la más utilizada por lo que para los adultos mayores esto se puede ver reflejado en una alta tasa de complicaciones e incluso mortalidad, por lo que individualizar cada caso, sus comorbilidades, riesgos pre, peri y postoperatorios, pueden llevar a disminuir estos números.

\section{Bibliografía}

1. Kärkkäinen JM, Acosta $S$. Acute mesenteric ischemia (part I) - Incidence, etiologies, and how to improve early diagnosis. Best Pract Res Clin Gastroenterol. 2017;31(1):15-25.

2. Khan SM, Emile SH, Wang Z, Agha MA. Diagnostic accuracy of hematological parameters in Acute mesenteric ischemia-A systematic review. Int J Surg [Internet]. 2019;66(February):18-27. Available from: https://doi.org/10.1016/j. ijsu.2019.04.005

3. Singh M, Long B, Koyfman A. Mesenteric Ischemia: A Deadly Miss. Emerg Med Clin North Am [Internet]. 2017;35(4):879-88. Available from: https://doi.org/10.1016/j.emc.2017.07.005

4. Solanas LL, Huguet MM. Enfermedades del mesenterio. Procesos inflamatorios. Patología vascular. Isquemia mesentérica. Med [Internet]. 2016;12(4):178-88. Available from: http://dx.doi. org/10.1016/j.med.2016.02.011

5. Motta-Ramírez GA, Sánchez-García JC, Ontiveros-Rodríguez A, López-Ramírez MA, Rebollo-Hurtado V, García-Ruiz A N-VH. Isquemia mesentérica aguda Acute mesenteric ischemia: an emergency which requires a comprehensive diagnostic approach. Scielo [Internet]. 2015;6688. Available from: http://webcir.org/revistavirtual/ articulos/2016/3_agosto/mex/isquemia_ mesenterica.pdf

6. Del Río Solá ML, González-Fajardo JA,

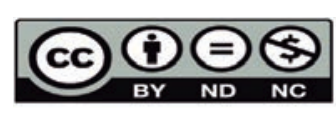


Vaquero Puerta C. Acute mesenteric ischemia. Diagnosis and treatment. Angiologia [Internet]. 2015;67(2):133-9. Available from: http://dx.doi. org/10.1016/j.angio.2014.05.015

7. Stone JR, Wilkins LR. Acute mesenteric ischemia. Tech Vasc Interv Radiol [Internet]. 2015;18(1):24-30. Available from: http://dx.doi. org/10.1053/j.tvir.2014.12.004

8. Clair DG, Beach JM. Mesenteric ischemia. N Engl J Med. 2016;374(10):959-68.

9. Lawson RM. Mesenteric Ischemia. Crit Care Nurs Clin North Am [Internet]. 2018;30(1):2939. Available from: https://doi.org/10.1016/j. cnc.2017.10.003

10. Herrero M, Agúndez I. Mesenteric ischaemia: Diagnosis and therapeutic algorithms. Angiologia [Internet]. 2017;69(1):34-40. Available from: http:// dx.doi.org/10.1016/j.angio.2016.06.002

11. Carver TW, Vora RS, Taneja A. Mesenteric Ischemia. Crit Care Clin [Internet]. 2016;32(2):15571. Available from: http://dx.doi.org/10.1016/j. ccc.2015.11.001

12. Nuzzo A, Maggiori $L$, Ronot $M$, Becq $A$, Plessier A, Gault N, et al. Predictive Factors of Intestinal Necrosis in Acute Mesenteric Ischemia: Prospective Study from an Intestinal Stroke Center. Am J Gastroenterol [Internet]. 2017;112(4):597605. Available from: http://dx.doi.org/10.1038/ ajg. 2017.38

13. Pereira Juliá A, Domingo Serrano F, Sánchez Ruiz S. Urgent patient care. Abdominal emergencies. Med [Internet]. 2019;12(87):508999. Available from: http://dx.doi.org/10.1016/j. med.2019.10.001

14. Mayo Ossorio MA, Pacheco García JM, Vázquez Gallego JM. Abdomen agudo. Med
[Internet]. 2016;12(7):363-79. Available from: http://dx.doi.org/10.1016/j.med.2016.03.012

15. David A Tendler, MDJ Thomas Lamont M. Overview of intestinal ischemia in adults. UpToDate [Internet]. 2018; Available from: https://www.uptodate.com/contents/overview-ofintestinal-ischemia-in-adults? search=isquemia mesentéricaaguda\& source=search result \& selectedTitle $=1 \sim 150 \&$ usage type=default\&display_rank=1\#H1822159668

16. Serracant Barrera A, Luna Aufroy A, Hidalgo Rosas JM, Cánovas Moreno G, FortuñoAndres JR, Falcó Fages J, et al. Isquemia mesentérica aguda: Utilidad de las técnicas de revascularización endovascular. Cir Esp. 2015;93(9):567-72.

17. Björck M, Orr N, Endean ED. Debate: Whether an endovascular-first strategy is the optimal approach for treating acute mesenteric ischemia. J Vasc Surg [Internet]. 2015;62(3):76772. Available from: http://dx.doi.org/10.1016/j. jvs.2015.04.431 